\title{
Pattern recognition with weighted complex networks
}

\author{
Jigger Cheh ${ }^{1, *}$ and Hong Zhao ${ }^{1,2}$ \\ ${ }^{1}$ Department of Physics, Institute of Theoretical Physics and Astrophysics, Xiamen University, Xiamen 361005, China \\ ${ }^{2}$ State Key Laboratory for Nonlinear Mechanics, Institute of Mechanics, Chinese Academy of Sciences, Beijing, 100080, China
}

(Received 15 May 2008; published 20 November 2008)

\begin{abstract}
In this paper we introduce a weighted complex networks model to investigate and recognize structures of patterns. The regular treating in pattern recognition models is to describe each pattern as a high-dimensional vector which however is insufficient to express the structural information. Thus, a number of methods are developed to extract the structural information, such as different feature extraction algorithms used in preprocessing steps, or the local receptive fields in convolutional networks. In our model, each pattern is attributed to a weighted complex network, whose topology represents the structure of that pattern. Based upon the training samples, we get several prototypal complex networks which could stand for the general structural characteristics of patterns in different categories. We use these prototypal networks to recognize the unknown patterns. It is an attempt to use complex networks in pattern recognition, and our result shows the potential for real-world pattern recognition. A spatial parameter is introduced to get the optimal recognition accuracy, and it remains constant insensitive to the amount of training samples. We have discussed the interesting properties of the prototypal networks. An approximate linear relation is found between the strength and color of vertexes, in which we could compare the structural difference between each category. We have visualized these prototypal networks to show that their topology indeed represents the common characteristics of patterns. We have also shown that the asymmetric strength distribution in these prototypal networks brings high robustness for recognition. Our study may cast a light on understanding the mechanism of the biologic neuronal systems in object recognition as well.
\end{abstract}

DOI: 10.1103/PhysRevE.78.056107

PACS number(s): 89.75.Hc, 42.30.Sy, 07.05.Mh

In spatial cognition of biologic neuronal systems, an object possesses many properties, such as color, size, brightness, and structures, etc. The main characteristic of an object is represented by its structures which is the primary reason to classify one object from other objects. The structure of an object is expressed as the description of the spatial arrangements and correlations among its individual parts [1-3]. It has been hypothesized that the aim of cortical information processing is to transform the highly redundant inputs into a higher-order representation which reveals the structure [4,5].

In the usual pattern recognition models, such as artificial neural networks [6-8], each pattern is attributed to a high dimensional vector which is often constructed by a row-byrow scan in the pixel box. Such expression is insufficient in expressing the structural information of each pattern, since the arrangements and correlations between the pixel points is not manifested in the vector. The synaptic matrix treats the computation of each dimension separately, and the spatial arrangements take no effect in computation. Thus, in order to overcome such shortcoming, a number of different methods are developed to extract the structural information within the raw inputs, such as feature extraction algorithms [9-11] used in preprocessing steps, or the local receptive fields in convolutional networks [12-14]. Different strategies are used in those methods. For example, a range of feature extraction algorithms are based upon sparse-coding representation which is motivated by evidence that sensory coding in the early stages of cortical information processing produces sparse representations. The local receptive fields in convolu-

\footnotetext{
*jk_jigger@xmu.edu.cn
}

tional networks are focused on the local connections to extract elementary features such as oriented edges, end points, or corners. Since the spatial correlations of each individual part of an object are strongly involved in the integration of information, from micro to macro, and vice versa, there are some papers [15-17] that indicate complex networks $[18,19]$ might be used in image characterization and classification in terms of the topology within the networks.

The idea of complex networks has been widely used in many different fields in the last decades. Complex networks intend to study the coupling architecture between different elements in a system and also the statistical behavior of that system. In complex networks, each element is treated as a vertex (or node, or point), and their interactions and correlations are represented as their connections (or links). For unweighted complex networks, the adjacency matrix describes the connections of vertexes, where 1 stands for a connection between two vertexes, and 0 stands for no connections. Recently, there is an increasing care to study weighted complex networks $[18,20,21]$, i.e., networks in which a real value is associated with each connection and the value describes the strength of that connection. This is most important in many cases since a complex topology is often associated with a large heterogeneity in the capacity and intensity of the connections, and unweighted complex networks are insufficient in describing such interactions. For example, the diversity of the predator-prey interactions in food chains is essential to the stability of the ecosystems [22,23]. In social networks, the strength of social contacts in order to characterize the correspondences is important to understand the relationship between people [24,25]. In many transportation networks, from the number of passengers in the airline traffic network [26] to the unequal traffic on the Internet [27], they are cru- 
cial quantities to study the topology and functions of these networks. Different capacities in the neuronal networks to transmit electric signals is the key to study how the neural system is able to process information. To study those networks, only weighted complex networks are capable to describe the diversity in the connections. For a weighted complex network, the adjacency matrix is now extended to weights matrix, the value of the weight is set to describe the strength of each different connection.

In our model, each pattern is attributed to a weighted complex network whose topology could represent its structure. The weighted complex network should express the spatial correlation between each individual pixel site to state the structure of an object. Usually, for one category, it has multiple patterns in the training database which share general structural characteristic across huge variations. Then we need to construct a prototype, which is also a weighted complex network here, for each individual category, and it could represent the general structural information of patterns in this category. In object recognition, it is called categorization [1]. So when an unknown pattern is presented, it would be described as a weighted complex network, and it is compared with the prototypal networks of different categories. If it is most similar with one definite prototypal network, then it is considered to be a pattern in that relative category.

So the key point is how to construct the weighted complex network for each pattern and each category, and how to compare the structural difference between two networks. For simplicity, here we consider the black and white patterns with binary pixel value, which are the most commonly used patterns in pattern recognition models, such as handwritten digits or print characters. As each plane pattern is centered in $k \times k$ pixel box, thereby its pixel matrix is $\hat{T}=\left\{T_{x . y} x\right.$ $=1, \ldots, k ; y=1, \ldots, k\}$ with $T_{x, y}= \pm 1$ where \pm 1 stand for the black and white pixels, respectively. In each pattern $\hat{T}$, the structural information contains two parts in the pixel sites: The pixel value $T_{x, y}$ and the relative pixel coordinates $(x, y)$. The regular treatment in pattern recognition is to transform the pixel matrix into a high-dimensional vector by row-byrow scanning, $\vec{v}=\left\{v_{i}=T_{x, y} \mid i=k(x-1)+y\right\}$. The pixel value of each pixel site is preserved, and the pixel coordinates are reordered by row-by-row scanning in such expression. The spatial information of each pattern is reparametrized here. Although the spatial information is maintained in such expression, the reordering does not consider the spatial correlations of the pixel sites, and the spatial arrangements of each pattern take no effect in computation since the computation of each dimension is separate. So this vector expression is insufficient in expressing the structural information of patterns. Figure 1(a) shows a pattern (digit 6) in the pixel box. The pixels with $T_{x, y}=1$ are denoted by solid dots there, while the pixels with $T_{x, y}=-1$ are denoted by the open dots. The pixel value and relative pixel coordinates together express the spatial arrangements of that pattern.

Our idea is to construct a weighted complex network to express patterns, and the pixel value and pixel coordinates of each pixel site and their spatial correlations are together considered. Each pixel site is attributed to a vertex in the (a)

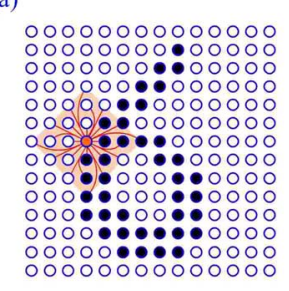

Pixel Box (b)

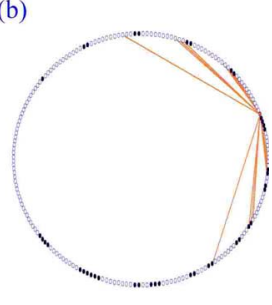

Complex Network
FIG. 1. (Color online) A sketch expressing the principle to construct the complex network. (a) A pattern (digit 6) in the pixel box. (b) It shows how a definite vertex gets its connections according to the pixel value and pixel coordinates of its relative pixel.

complex networks. In the complex networks, each vertex contains a color value $c_{i}$ which equals the pixel value in the relative pixel site. The connections and connection weights between vertexes is constructed to reveal the spatial arrangements of pixel sites according to their coordinates. Since there are $k \times k$ pixel sites, thus there are $k \times k$ vertexes in the complex networks. The relative $k^{2} \times k^{2}$ weights matrix $\hat{w}=\left\{w_{i, j} \mid i=k\left(x_{1}-1\right)+y_{1}, j=k\left(x_{2}-1\right)+y_{2}\right\}$ is constructed as follows:

$$
w_{i, j}= \begin{cases}T_{x_{1}, y_{1}} \times T_{x_{2}, y_{2},}, & \left|x_{1}-x_{2}\right|+\left|y_{1}-y_{2}\right|<\lambda, \\ 0, & \left|x_{1}-x_{2}\right|+\left|y_{1}-y_{2}\right| \geqq \lambda, \\ 0, & \left|x_{1}-x_{2}\right|+\left|y_{1}-y_{2}\right|=0 .\end{cases}
$$

Figures 1(a) and 1(b) show how the weighted complex network is constructed according to the pixel value and pixel coordinates of a definite pattern. Through the definition in (1), the spatial correlations among two different pixel sites $i$ and $j$, is described by their connection weights according to their pixel value and pixel coordinates now. Such representation will remain the structural information of patterns in the topology of the complex networks.

For this complex network, the number of the vertexes is $M_{1}=k^{2}$, and since it is a symmetric complex network without self-connection $\left(w_{i, j}=0\right.$ if $\left.i=j\right)$, the number of connections in this network is $M_{2}=\frac{1}{2} k^{2}\left(k^{2}-1\right)$. According to the weights matrix, we can get the maximum number of nonzero connections of a vertex, $P_{\max }=4 \lambda+4 \sum_{i=1}^{\lambda-1}(\lambda-i)$, and the minimum number of nonzero connections of a vertex, $P_{\min }=2 \lambda$ $+\sum_{i=1}^{\lambda-1}(\lambda-i)$. So the number of nonzero connections of a vertex $P$ is

$$
\frac{1}{2} \lambda^{2}+\frac{3}{2} \lambda \leqq P \leqq 2 \lambda^{2}+2 \lambda .
$$

The number of nonzero connections in this complex network is $M_{2}^{\prime}=\frac{1}{2} k^{2} \bar{P}$. As it shows in formula (2), the nonzero connection of a vertex is restricted by the spatial parameter $\lambda$, so if $\lambda \ll k^{2}$ the connections in the complex networks would be very sparse. For example, $\lambda=6$ is the nonzero connection of a vertex $27 \leqq P \leqq 84$ and $k^{2}=28 \times 28=784, \bar{P} \ll k^{2}-1 \sim k^{2}$ and the nonzero connections in the complex network is $M_{2}^{\prime}$ $\ll M_{2}$. Later, it shows that $\lambda=6$ is correct for the optimal parameter for recognition. Since only the nonzero connections in the complex networks would be in effect in compu- 
tation and determines the topology of the network, the sparse connected networks need much less computation time and memory than fully connected networks.

Here we have introduced a spatial parameter $\lambda$ to get the connection weights between pairwise vertexes. It is a controllable parameter that can be selected empirically to get the maximum accuracy ratio in recognition. It describes the influence range of a pixel site, if another pixel site is far away from it, the connection between them reduces to zero. Too large or too small influence range will decrease the accuracy ratio, as it shall be seen later, the maximum accuracy ratio always occurs on the same medium value. As shown in Fig. 1(a), we illustrate a pixel site (with orange dots) and its correlations (the orange lines which connect other pixel sites in the pink box). The influence range of that pixel site is cutoff by $\lambda=4$. Figure 1 (b) shows the relative orange vertex connects with other vertexes whose relative pixel sites are in the influence range in the pixel box. The spatial parameter will determine whether a vertex in the complex network would connect with the orange vertex.

The correlation between two pixel sites is expressed by multiplying their pixel value. For binary pixel value here, +1 stands for the black pixel and -1 stands for the white pixel, we have $1 \times 1=(-1) \times(-1)$ and $1 \times(-1)=(-1) \times 1$. The correlation between two pixel sites with the same pixel value is 1 , and with different pixel value is -1 . So even if we reverse the color of that pattern, the weights between the relative vertexes in the complex network remain the same. It states a simple principle, whether the pattern is a black diagram on a white background, or white diagram on a black background, the structure of it stays the same. The vector expressing method cannot reveal this principle.

Here each vertex has a color value $c_{i}$ to state the pixel value of its relative pixel site. In complex networks, a measure is often given to a vertex to qualify its function. For example, betweenness $[28,29]$ may state the importance of an individual in social networks, or the centrality $[18,30]$ may measure the frequency of a vertex being visited by random walkers.

The prototypal network of a category is meant to represent the general structural characteristic of patterns in that category. It neglects the variation of each individual pattern, and only their common structural information should be expressed. Since those different patterns have no priority of importance in that category, thus the simplest way to construct the prototypal network is by averaging their weights matrices. In the same way, we also obtain the vertex color of the prototypal network. Such method can be improved with Monte Carlo adaptation rule via the neural networks method $[7,8]$, which would however take much more time in training. Here $N$ denotes the $N$ th category, and $n$ denotes the amount of training patterns in this category.

$$
\begin{aligned}
w_{i, j}^{(N)} & =\frac{\sum w_{i, j}^{(n)}}{\sum n}, \\
c_{i}^{(N)} & =\frac{\sum c_{i}^{(n)}}{\sum n} .
\end{aligned}
$$

As it shall be seen later (Figs. 3 and 4), in the prototypal complex networks, the clustered structures and asymmetric strength distributions come naturally. The clustered structure reveals the structure of that category, and the asymmetric strength distribution reveals the different roles of vertexes in the networks.

To classify the category of an unknown pattern, we need to calculate the structural differences between the unknown pattern and the prototypes. The category of the unknown pattern is selected to be the one whose prototypal network has the minimum structural difference. The weights matrix of the unknown pattern is $w_{i, j}$, the weights matrix of the $N$ th category is $w_{i, j}^{(N)}$, so the structural difference $\Delta^{(N)}$ is calculated as follows:

$$
\Delta^{(N)}=\sum_{i}\left(\sum_{j}\left(w_{i, j}-w_{i, j}^{(N)}\right)^{2}\left|\left(c_{i}-c_{i}^{(N)}\right)\right|\right) .
$$

In formula (3), the component $\left|\left(c_{i}-c_{i}^{(N)}\right)\right|$ denotes the color differences of vertexes between the unknown pattern and the $N$ th category. If we neglect the color information and only consider the structure, we delete $\left|\left(c_{i}-c_{i}^{(N)}\right)\right|$ in this formula, then there is no discrimination between whether it is black diagrams on a white background, or white diagrams on a black background. We may use such formula to recognize the reversed colored patterns as well. It shall be seen later, the main information for recognition is in the structures rather than the colors.

Our model can be improved if one adds a neural classifier after the complex networks layer. Instead of using prototypal networks and calculate the structural difference between an unknown pattern and the prototypes, the neural networks layer would be used to recognize and classify the patterns which now are represented as complex networks that describes the correlations between the pixel sites and their spatial arrangements. There are some attempts to integrate complex networks and neural networks together [31,32]. Their idea is to add the adjacency matrix to modulate the synaptic matrix of the neural networks, and study how the different topology of complex networks affects the functions of neural networks. Our work may inspire another way to integrate complex networks and neural networks together, in which complex networks layer intends to describe spatial correlated patterns, and neural networks layer intends to classify those complex networks. However, here our attention is only focused on the complex networks representation which describes the structure of the patterns, and study the interesting properties within those complex networks. In doing so, the computation time cost and memory cost would be much less to get the same result than using some neural classifiers.

To verify and test our model, we apply it to recognize the handwritten digits in the MNIST database [33] as an example. The database is a subset of the NIST database which is constructed from real-world digits written by Census $\mathrm{Bu}-$ reau employees and high-school students. It contains a training set of 60000 samples, and a testing set of 10000 samples. The digits are centered in $28 \times 28$ pixel boxes. For simplicity, we neglect the grey levels to reduce them to black digits written on white backgrounds. 


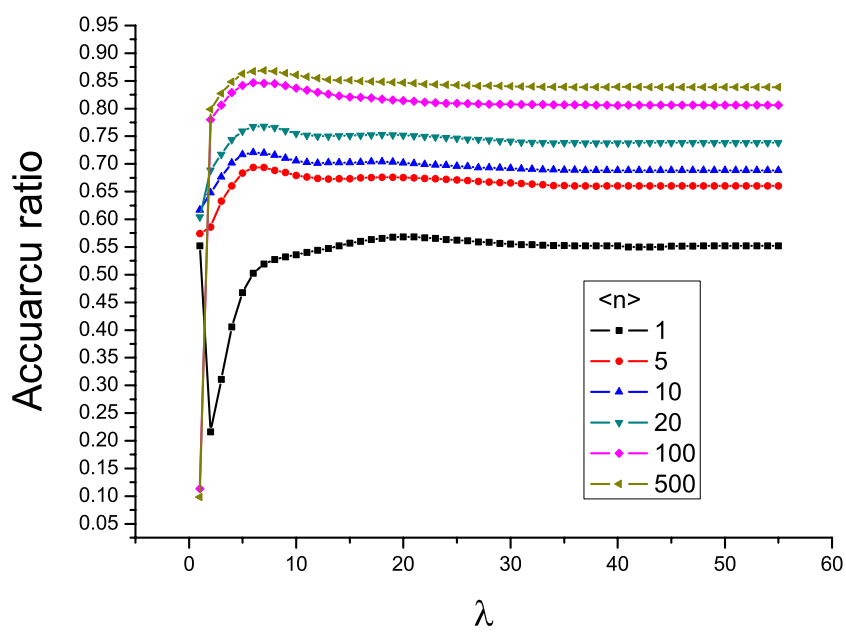

FIG. 2. (Color online) The accuracy ratio varies with different spatial parameters $\lambda$. Each category averages $n$ training samples from 1 to 500, and the spatial parameter $\lambda$ varies from 1 (all of the vertexes are isolated) to 55 (all of the vertexes are connected).

Based on the 60000 training samples to recognize the 10000 testing samples, the maximum accuracy ratio reaches more than $93 \%$ with six prototypes per category. If we only consider the structures, the maximum accuracy ratio reaches 91\%. This states that structures, not the color, are the key to distinguishing different patterns. Whether it is either a black graph on a white background or a white graph on a black background, the structure rather than the colors determines the pivotal information of that pattern. The result shows the potential to apply our model in real-world pattern recognition.

The accuracy varies with different spatial parameter $\lambda$ in recognition. As shown in Fig. 2, the accuracy peak always occurs on the same medium value when averaging more than one pattern. It has nothing to do with the amount of patterns it averages. Either too large or too small value will decrease the recognition accuracy ratio. As stated in the foregoing, the spatial parameter limits the connections of a vertex in the networks, according to the range of nearest neighbors of its relative pixel site. The optimal connection number of each vertex remains constant in recognition, it is insensitive to the networks. It is similar to the phenomena observed in the Hippocampal interneuronal network $[18,34]$, where the average number of synaptic contacts per cell is also constant insensitive to the networks. The reason why the optimal connection number is constant may come from the dynamical process in the network, and it needs to be verified in the future.

The strength of the weighted complex networks $[18,20,21]$ is defined as follows:

$$
s_{i}^{(N)}=\sum_{j=1}^{M_{1}} w_{i, j}^{(N)} .
$$

The strength of a vertex integrates the information about its connectivity and the weights of the connections, and can be considered as the natural generalization of the connectivity.

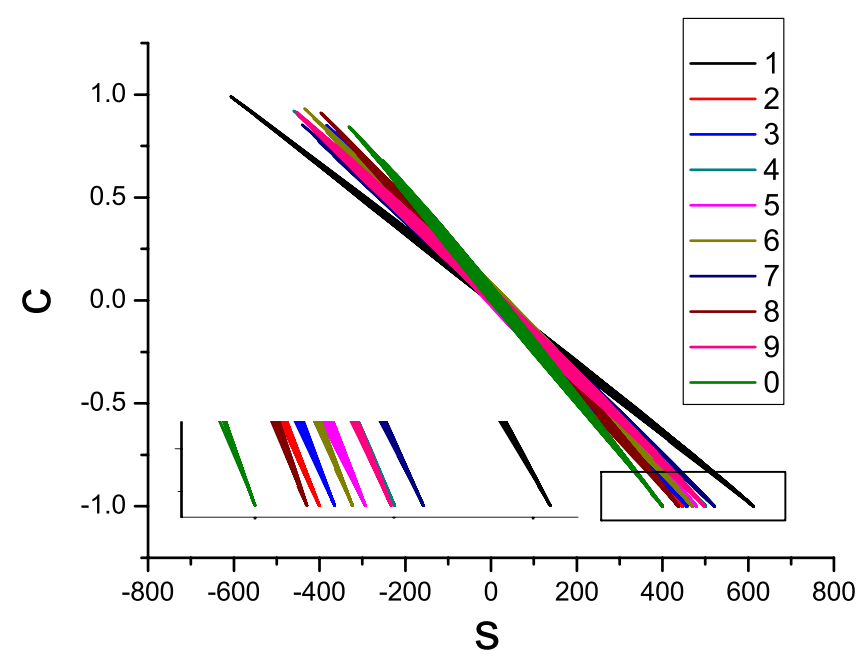

FIG. 3. (Color online) The relation between strength and color of each vertex in the complex networks.

It is the natural extension of degree $k[18,20,21]$ defined in unweighted complex networks.

If we let the spatial parameter $\lambda \geqq 54$ so that all vertexes are connected in the complex networks, we get an approximate linear relationship between the strength and color of each vertex in the prototypal networks (Fig. 3).

The approximate linear relation states the general properties of patterns in each category, thus it provides us a way to visually compare the topological similarity of each category in this graph. The categories of digits are $0,8,2,3,6,5,9,4$, 7, 1 from the left-hand to the right-hand side, down to the bottom. We can obtain that the digits 9 and 4 are more similar than any other digits, and the digits 0 and 1 are the most different in these categories. Such result indeed accords with our empirical experience as well. The approximate linear relation also indicates that the tendency of vertex with strong strength is apt to have negative color value, and vice versa.

We visualize the prototypal networks to see their connections, and to see whether their topology reveals the general
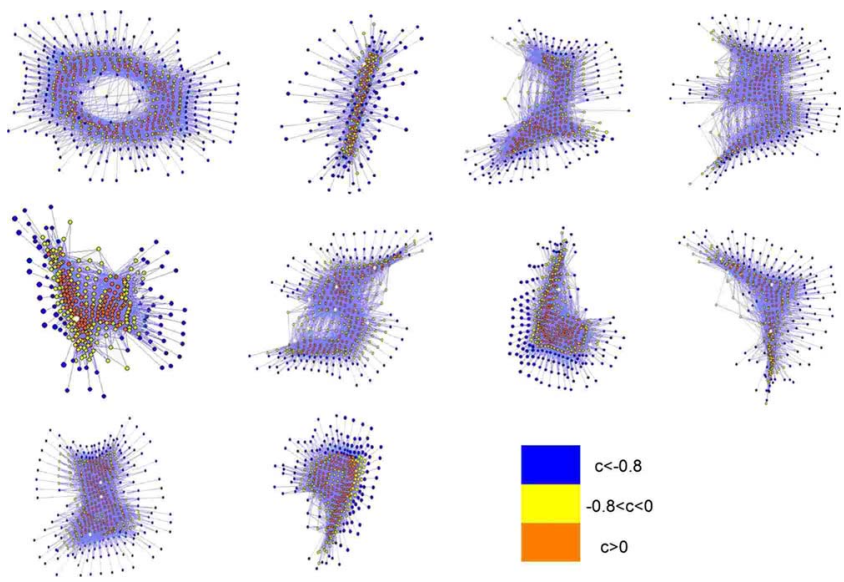

FIG. 4. (Color online) The clustered structure of the 10 prototypal networks. We let each nonzero weights value add 1.5 to lay out the networks with similarity weights. The color of each vertex also is marked. For clarity the connections between vertexes with negative weights are hidden. 


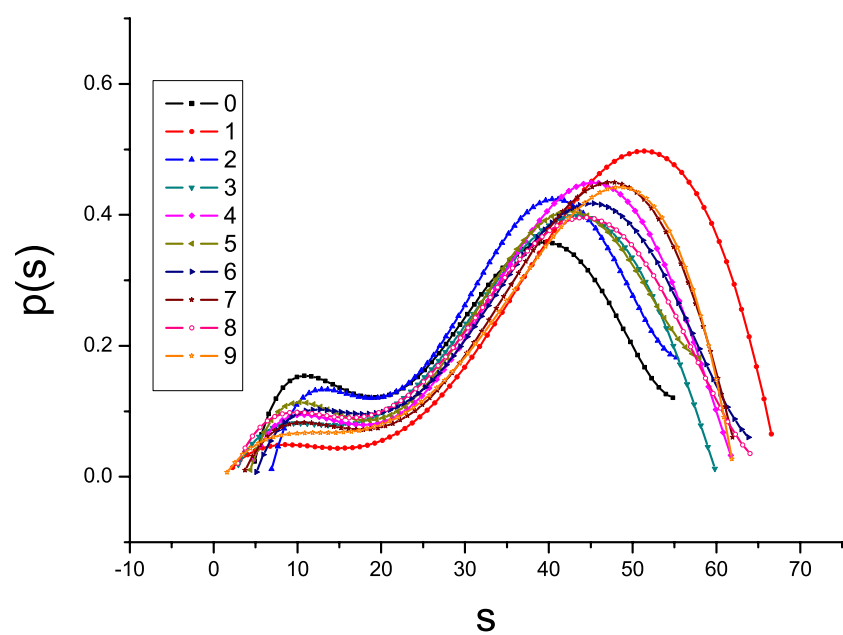

FIG. 5. (Color online) The strength distribution of the 10 prototypal networks. The precision of strength is $80 \%$, that if a vertex with strength $s_{2}$ and $\frac{\left|s_{1}-s_{2}\right|}{s_{1}}<0.8$, then we label it as the vertex with strength $s_{1}$ as well. Different curve stands for different category, from digit 0 to digit 9 .

structural information of each category. The Pajek program for large network analysis is used for visualization here. As shown in Fig. 4, the clustered structures appear naturally in the prototypal networks.

These clustered structures indeed reveal the structures of handwritten digits of the 10 different categories, from digit 0 to digit 9. The color parameter also helps in reconstructing the structures of different categories. In biology, there are a lot of physiological experiments indicating that neuronal systems display such clustered structures as well, such as in cat visual cortex and human functional brain networks [35-37]. However, the origin of such clustered structures is still unexplained. Our study may provide a possible explanation to understand that mechanism, where the clustered structure of neuronal system comes from the structure of the knowledge it learns. Since the information the neuronal system receives is clustered with a definite structure, so that in evolution the neuronal structure would finally reveal such structure.

To investigate the connections and hierarchy of each vertex in the prototypal networks, we consider the strength distribution here. The strength of a vertex integrates the information about its connectivity and the weights of its links. The strength distributions of the prototypal networks are shown in Fig. 5, where $p(s)$ denotes the fraction of vertexes with strength $s$ in the 10 prototypal networks. Their distributions are similar in that there are two peaks with different heights in the curves.

Such kind of asymmetric distribution is very interesting, because usually the distribution is either exponential distribution with only one peak, or power-law distribution without any peaks. The reason why there are two distribution peaks can be explained, such that there are two kinds of different roles of vertexes in those networks. A vertex is either a diagram vertex or a background vertex, and it is the diagram shown in the background. The diagram vertex would have a small strength value, since it is mainly surrounded by the background vertexes with minus weights. The first peak is formed by their strength distribution. Equally, the second peak is formed by the strength distribution of the background vertexes as well.

The diagram vertexes with smaller weights are more important than the background vertexes in recognition. Such asymmetric distribution would enable the prototypal networks to be resistant to random failures $[18,30,38]$ as the scale-free distribution. To show the asymmetric distribution indeed brings high robustness in the prototypal networks, we destroy the connections between the background vertexes (let $w_{i, j}=0$ if both $c_{i}$ and $c_{j}$ are negative) in the prototypal networks previously used in recognition. In doing so, about $30 \%$ vertexes have been wiped out and $85 \%$ vertexes are more or less affected. The connections of the wiped out vertexes are all cut off which makes those vertexes take no effect in recognition. Only $15 \%$ vertexes remain with their connections intact after such destruction. However, the accuracy ratio only decreases form $93 \%$ to $90 \%$, so that the primary function of those networks still remains. It is similar in the biologic neuronal systems, that they could keep the same, through degraded function after suffering the destruction of many neurons and their connections [35,39].

Here we have shown the basic properties of using the complex networks in patterns recognition. In this model, each pattern is attributed to a weighted complex network to express its structure. A prototypal network is constructed across multiple patterns in the same category, and it represents their common structural characteristics. These prototypal networks are used to recognize an unknown pattern. As an example, we apply this model to recognize the handwritten digits, and the result shows the potential for real-world application. A controllable spatial parameter is introduced to get the optimal recognition accuracy, and it remains constant insensitive to the amount of training samples. We have discussed the interesting properties shown in the prototypal networks. An approximate linear relation is found between the strength and color of vertexes, and we can use this relation to compare the common topological similarity between different categories. The clustered structure of the prototypal network reveals the structure of its relative category. The strength distribution possesses two asymmetric peaks, and such distribution brings high robustness in the prototypal networks. Our model may also provide us with some new sights to understand the structures and mechanism of biologic neuronal systems. We hope our work will further stimulate interdisciplinary studies in complex networks.

The authors thank Ping Hwang for useful discussions and Yue Zhang for help with the preparation of the paper. This research was partly supported by National Basic Research Program of China (973 program) (Contract No. 2007CB814800), and National Natural Science Foundation of China (Contract No. 10475067). 
[1] M. Riesenhuber and T. Poggio, Nat. Neurosci. 3, 1199 (2000).

[2] I. Biederman, Psychol. Rev. 94, 115 (1987).

[3] J. Hummel and I. Biederman, Psychol. Rev. 99, 480 (1992).

[4] H. B. Barlow, Perception 1, 371 (1972).

[5] H. B. Barlow, Neural Comput. 1, 295 (1989).

[6] S. Haykin, Neural Networks: A Comprehensive Foundation (Prentice-Hall, New Jersey, 1999).

[7] H. Zhao, Phys. Rev. E 70, 066137 (2004).

[8] T. Jin and H. Zhao, Phys. Rev. E 72, 066111 (2005).

[9] R. Steinert, M. Rehn, and A. Lansner, in ESANN'2006 Proceedings-European Symposium on Artificial Neural Networks, 2006, pp. 161-166.

[10] A. J. Bell and T. J. Sejnowski, Adv. Neural Inf. Process. Syst. 9, 831 (1996)

[11] P. Foldiak, Biol. Cybern. 64, 165 (1990).

[12] Y. LeCun, L. Bottou, Y. Bengio, and P. Haffner, Proc. IEEE 86, 2278 (1998).

[13] B. J. Balas and P. Sinha, Neural Comput. 18, 497 (2006).

[14] F. Alleau, E. Poisson, C. V. Gaudin, and P. LeCallet, Proceedings of the 2003 Joint Conference of the Fourth International Conference on Information, Communications and Signal Processing, 2003 and the Fourth Pacific Rim Conference on Multimedia, 2004, Vol. 2, pp. 989-993.

[15] L. da F. Costa, e-print arXiv:cond-mat/0606060.

[16] L. Diambra and L. da F. Costa, Bioinformatics 21, 3846 (2005).

[17] T. Chalumeau, L. da F. Costa, O. Laligant, and F. Meriaudeau, Proc. SPIE 6356, 63561E (2007).

[18] S. Boccaletti, V. Latora, Y. Moreno, M. Chavez, and D.-U. Hwang, Phys. Rep. 424, 175 (2006).

[19] S. H. Strogatz, Nature (London) 410, 268 (2001).

[20] S. H. Yook, H. Jeong, A.-L. Barabasi, and Y. Tu, Phys. Rev. Lett. 86, 5835 (2001).
[21] A. Barrat, M. Barthelemy, and A. Vespignani, Phys. Rev. Lett. 92, 228701 (2004).

[22] A. E. Krause, K. A. Frank, D. M. Mason, R. E. Ulanowicz, and W. W. Taylor, Nature (London) 426, 282 (2003).

[23] S. L. Pimm, Food Webs (University of Chicago Press, Chicago, 2002).

[24] M. Granovetter, Am. J. Sociol. 78, 1360 (1973).

[25] R. L. Breiger, Annu. Rev. Sociol. 21, 115 (1995).

[26] L. A. N. Amaral, A. Scala, M. Barthelemy, and H. E. Stanley, Proc. Natl. Acad. Sci. U.S.A. 97, 11149 (2000).

[27] R. Pastor-Satorras and A. Vespignani, Evolution of Networks: From Biological Nets to the Internet and WWW (Oxford University Press, Oxford, 2003).

[28] J. Scott, Social Network Analysis: A Handbook (Sage, London, 2000).

[29] S. Wasserman and K. Faust, Social Network Analysis (Cambridge University Press, Cambridge, 1992).

[30] A. E. Motter, Phys. Rev. Lett. 93, 098701 (2004).

[31] J. J. Torres, M. A. Munoz, J. Marro, and P. L. Garrido, Neurocomputing 58-60, 229 (2004).

[32] J. Lu, J. He, J. Cao, and Z. Gao, Phys. Lett. A 354, 335 (2006).

[33] Y. LeCun, The MNIST database of handwritten digits, http:// yann.lecun.com/exdb/mnist/index.html.

[34] G. Buzskai and X.-J. Wang, J. Neurosci. 15, 6402 (1996).

[35] J.-P. Eckmann, O. Feinerman, L. Gruendlinger, E. Moses, J. Soriano, and T. Tlusty, Phys. Rep. 449, 54 (2007).

[36] T. Binzegger, R. J. Douglas, and K. A. C. Martin, J. Neurosci. 24, 8441 (2004).

[37] O. Sporns, D. R. Chialvo, M. Kaiser, and C. C. Hilgetag, Trends Cogn. Sci. 8, 418 (2004).

[38] R. Albert, H. Jeong and A.-L. Barabasi, Nature (London) 406, 378 (2000).

[39] J. M. Fearnley and A. J. Lees, Brain 114, 2283 (1991). 